\title{
Stress Analysis for Nanomembranes under Stamp Compression
}

\author{
Shutao Qiao ${ }^{\mathrm{a}}$, Nanshu $\mathrm{Lu}^{\mathrm{a}, \mathrm{b}, \mathrm{c}^{*}}$ \\ ${ }^{a}$ Center for Mechanics of Solids, Structures and Materials, Department of Aerospace \\ Engineering and Engineering Mechanics, NASCENT Center, ${ }^{\mathrm{b}}$ Department of Biomedical \\ Engineering, ${ }^{\mathrm{c}}$ Texas Materials Institute, the University of Texas at Austin, Austin, Texas \\ 78712, US.
}

\begin{abstract}
Many high performance flexible and stretchable electronics are manufactured by transferring inorganic semiconductor nanomembranes from their rigid donor substrates to soft receiving substrates via elastomeric rubber stamps. As nanomembrane thickness reduces to nanometers or subnanometers (e.g., 2D materials), they can be easily ruptured during the stamping process by shear stresses. Through analytical modeling, this paper reveals the membrane stress in the nanomembrane induced by stamp compression as a function of the stamp and nanomembrane property and geometry, as well as the tractionseparation relation between the nanomembrane and the donor substrate. While membrane stress in the nanomembrane increases monotonically with the compressive loading applied on the stamp, an abrupt increase appears when nanomembrane-substrate interface starts to fail. While the stamp is assumed to be incompressible material in the main text, more general solutions for compressible stamps are offered in the supplementary material.
\end{abstract}

Keywords: nanomembrane; transfer; stamp

${ }^{*}$ Corresponding author. Tel.:+1 512-471-4208Ｅmail: nanshulu@utexas.edu

Address: 210 E. 24th St, Austin, TX 78712 


\section{Introduction}

Inorganic semiconductors such as silicon ( $\mathrm{Si})$, gallium arsenide (GaAs), and gallium nitride $(\mathrm{GaN})$ are, by far, the most well-established materials for high performance electronics and optoelectronics. Although these materials are intrinsically rigid and brittle, when exploited in mechanically optimized layouts, they can be integrated on soft polymer supports to yield integrated flexible or stretchable functional devices. The result is an electronics/optoelectronics technology that offers the performance of conventional wafer-based systems, but with the mechanics of a piece of paper or a rubber band. Examples include flexible displays [1], solar cells [2, 3], stretchable optoelectronics [4] and photovoltaics [5], as well as bio-inspired [6, 7] and bio-integrated [8-10] electronics.

Semiconductors in these examples are in the form of nanomembranes (NMs) and nanoribbons (NRs) due to their ultra-low flexural rigidities and small strains even when bent or buckled to small radii of curvature. Since high quality crystalline NMs are usually formed by wafer bonding and polishing or controlled fracture [11], or by epitaxial growth on crystalline substrates $[3,12]$, they need to be integrated onto substrates of choice in a controlled, deterministic fashion by the techniques of transfer printing [13-15]. An important feature of this process is that it exploits the known, lithographically defined positions and orientations of undercut-etched nanostructures. As a common example, Si NMs can be formed by releasing the top Si layer of a silicon-on-insulator (SOI) substrate after etching away the buried oxide layer with hydrofluoric acid [16, 17]. Other SOI-like structures that can serve as routes to different semiconductor nanomaterials include germanium-on-insulator (GOI), 
silicon-germanium-on-insulator (SGOI), as well as III-V semiconductors and many other combinations [18-21]. Retrieval from these predetermined sites, followed by release onto other substrates, can be accomplished with rubber stamps such as the polydimethylsiloxane (PDMS). The procedure involves switching the strength of adhesion between these structures and PDMS from strong to weak states by exploiting viscoelastic and/or geometric effects [13, 14, 22, 23].

Although transfer printing has been proved effective for many semiconductor NMs, the difficulty escalates when NM thickness drops below $100 \mathrm{~nm}$, which gains optical transparency [24] and biodegradability [25] in the case of Si NM. Cracks and wrinkles easily form in ultrathin NMs picked up by PDMS stamps from our own experimental experience. This is why transparent thin film transistors (TFTs) based on Si NMs have been rarely reported and state-of-the-art transient or biodegradable electronics [26, 27] are still using $\mathrm{Si} \mathrm{NMs}$ with thickness in the hundreds of nanometers regime. Similar problems exist when peeling or exfoliating nanometer or subnamometer thick two-dimensional (2D) atomic layers using PDMS stamps. In our recent paper [28], few layer molybdenum disulfide $\left(\mathrm{MoS}_{2}\right)$ flakes were exfoliated from a synthetic $\mathrm{MoS}_{2}$ crystal by either adhesive tapes or PDMS stamps. In either case, cracks, wrinkles, and buckle delaminations are clearly visible in exfoliated samples.

So far, the cause of failure when stamping on ultrathin NMs remains unclear and it hence lacks a guideline for minimizing stamping induced NM failure. To tackle this problem, we decide to first find out the stamp-NM interaction and then set up a boundary value problem (BVP) to solve for the membrane stress developed in the 
NM, as described in Section 2. Our results reveal that the compressive load applied on the stamp plays a significant role. The size and mechanical properties of the stamp can affect the stamp-NM interaction and can be used to tilt the membrane stress to avoid cracking. Results are summarized in Section 3 and discussed in Section 4 with manufacturing guidelines provided.

\section{Boundary Value Problem}

The stamping process with an unstructured, flat stamp is illustrated by crosssectional schematics in Figs. 1a \& 1b. First, the PDMS stamp backed by a rigid layer (e.g., a glass slide) is making a gentle contact on the NM which is sitting on its donor substrate (e.g., Si NM on undercut oxide or chemical vapor deposited (CVD) graphene on seed copper (Cu)) (Fig. 1a). Then an external compression characterized by the compressive strain $\epsilon$ is applied to the stamp to form an intimate contact with the NM such that the NM can later be peeled off from the donor substrate by the stamp. Due to the Poisson's effect, the vertically compressed stamp would expand laterally, resulting in shear stresses on the stamp-NM interface [29-31], which rubs the NM and may eventually lead to cracked NM (Fig. 1b).

Assuming the stamp is an infinitely long strip into the paper, Fig. 1c draws the stresses experienced by the NM in a 2D plane strain model. The shear stress applied by the stamp on the top surface of the NM is labeled as $\tau_{t o p}$ whereas the shear stress applied by the donor substrate on the bottom surface of the NM is labeled as $\tau_{\text {bottom }}$. A free body diagram (FBD) for the boxed part of the NM is provided as an inset in 
Fig. 1c, which suggests that the edge on the right is a traction free surface while the membrane stress in the NM at the cut can be given by the equilibrium condition:

\begin{tabular}{|l|l|}
\hline$\sigma(x)=\frac{\int_{x}^{a}\left(\tau_{\text {top }}(\eta)-\tau_{\text {bottom }}(\eta)\right) d \eta}{h_{m}}$ & (1) \\
\hline
\end{tabular}

where $x$ starts form the middle of the NM (Fig. 1a), $a$ is the half size of the stamp and $\mathrm{NM}$ and $h_{m}$ is the thickness of the NM. Equation (1) offers a simple explanation why ultrathin NMs are more prone to fracture during stamping: as the membrane stress is inversely proportional to the NM thickness, when NM thickness drops from hundreds of nanometers to few nanometers, the membrane stress can be elevated by hundred times. Given $h_{m}$, the central goal of this paper is to quantify $\sigma(x)$ as a function of the stamp geometry and property, the NM-substrate interface property, as well as the compressive load $\epsilon$ applied on the stamp.

$\tau_{\text {bottom }}$ is the shear stress developed to balance $\tau_{\text {top }}$. Before discussing $\tau_{\text {top }}$, we assume that the evolution of $\tau_{\text {bottom }}$ has to follow the shear traction-separation relation (TSR) along the NM-substrate interface. Various TSRs have been developed and applied to describe physical phenomena of interface fracture. For simplicity, a rectangular TSR as shown in Fig. 1d is adopted, where $\tau_{0}$ is the NM-substrate interface adhesion strength, $\delta_{c}$ is the critical separation between NM and substrate, and the area within this rectangle is the interface adhesion energy. As the substrate is assumed rigid and non-deformable, separation between the NM and the substrate is identical to the NM displacement in the $x$ direction, $u(x)$. Whenever $|u(x)|$ exceeds $\delta_{c}$, the NM will fully and permanently detach from the substrate at that point and 
there is no more interaction between them. Re-adhesion is not allowed in this model. Hence $\tau_{\text {bottom }}$, a function of $u(x)$, could be written as

$\tau_{\text {bottom }}=\left\{\begin{array}{rr}0 \sim \tau_{0} & |u(x)|=0 \\ \tau_{0} & 0<|u(x)|<\delta_{c} \\ 0 & |u(x)| \geq \delta_{c}\end{array}\right.$

$\tau_{\text {top }}$ captures the interaction between the stamp and the NM. Since the rubber stamp is sandwiched between the rigid backing layer and the rigid NM-on-substrate and the bottom of the stamp can be assumed fixed due to minimum deformation in both the lateral and vertical direction of the stiff NM. Thus, we can adopt our previous solutions for an infinitely long elastic layer bonded and compressed between two rigid plates (Eqs. (44) and (48) in [29]):

$\tau_{t o p}(x)=\epsilon A \sinh \left(\alpha \frac{x}{h}\right)$

where $A$ and $\alpha$, determined by the mechanical properties and geometry of the stamp, are given by the following equations

\begin{tabular}{|l|l|l|}
\hline$A=\frac{\alpha v E}{2(1+v)(1-2 v) \cosh \left(\frac{\alpha a}{h}\right)}$ & (4) \\
\hline
\end{tabular}

where $E$ and $v$ are the Young's modulus and Poisson's ratio of the stamp material, $h$ is the stamp thickness, and $\alpha$ is the positive root of Eq. (5):

$\alpha=(3-4 v) \sin (\alpha)(-1 \leq v<1 / 2)$

Figures 1e \& 1f demonstrate the effects of stamp aspect ratio $(a / h)$ and Poisson's ratio $(v)$, respectively, with $\tau_{\text {top }}$ normalized by $E \epsilon$. In Fig. 1e, with a given Poisson's ratio $(v=0.45)$ but different aspect ratios of the stamp (red curve: $a / h=8$ vs. black curve: $a / h=10$ ), both interfacial shear stresses are monotonically increasing from 
zero at the center of the stamp towards the edge of the stamp. This observation suggests that if the NM is much smaller than the stamp and is located towards the center of the stamp, the shear stress applied on the NM can be minimal. Moreover, since the stamp with higher aspect ratio generates lower shear stress, we can increase the lateral dimension of the stamp to further reduce the shear stress experienced by the NM. However, such effect of aspect ratio vanishes if the stamp is made of strictly incompressible material as discussed in the next paragraph. Hence the strategy of enlarging stamp aspect ratio to lower $\tau_{\text {top }}$ does not work for incompressible stamps.

In Fig. 1f, the stamp aspect ratio $a / h$ is set to be 5 while the Poisson's ratio changes from 0.3 to 0.5 . The incompressible solution $(v=0.5)$ is achieved by taking the limit $v \rightarrow 0.5$ in Eqs. (3)-(5), which yields $\alpha \rightarrow 0, A \rightarrow \infty, A \sinh (\alpha x / h) \rightarrow$ $2 E x / h$, and hence:

$\tau_{\text {top }}(x)=2 E \epsilon \frac{x}{h}$

which recovers the linear interfacial shear stress distribution observed in Gent's experiments for compressed rubber blocks [31]. According to Fig. 1f, stamps with smaller Poisson's ratio generate lower $\tau_{\text {top }}$ due to weaker Poisson's effect, which agrees with our intuition. A straightforward strategy following this result is that materials with smaller Poisson's ratio are preferred stamp materials when stamping on the NMs. However, in most cases, PDMS, which is perfectly incompressible, are used as stamps for transfer printing due to their desirable surface energy and moldability. So from now on, we will just focus on incompressible stamps with $\tau_{\text {top }}$ given by Eq. (6) in the following main text. More general solutions and results for 
stamps made out of compressible materials, with $\tau_{\text {top }}$ given by Eq. (3), are summarized in the supplementary material.

Neglecting the deformation in the thickness direction of the NM, the constitutive relation between the membrane stress and displacement is given by

$$
\sigma(x)=\left(2 \mu_{m}+\lambda_{m}\right) \frac{d u}{d x}
$$

where $\mu_{m}$ and $\lambda_{m}$ are the two Lamé constants of the NM material. Plugging Eq. (7) into Eq. (1) and then taking the first order derivative yield:

$$
\frac{d^{2} u(x)}{d x^{2}}+\frac{\tau_{t o p}(x)-\tau_{\text {bottom }}\left(u_{x}(x)\right)}{\left(2 \mu_{m}+\lambda_{m}\right) h_{m}}=0
$$

With boundary conditions $(\mathrm{BCs})$ defined as $u(0)=0$ due to symmetry and $u^{\prime}(a)=0$ due to the traction free edge, we have established a BVP for the NM. The solution to the $2^{\text {nd }}$ order ODE of Eq. (8) is the lateral displacement $u(x)$ of the NM. Once $u(x)$ is solved, the membrane stress can be readily obtained through Eq. (7).

\section{Solutions}

The BVP in Section 2 can be solved analytically with parameters that characterize $\tau_{\text {top }}$ and $\tau_{\text {bottom }}$ given by Eqs. (3) and (6). Assuming that the compressive load on the stamp increases quasi-statically, different solutions of Eq. (8) can be obtained through the following segmental analysis:

When $\epsilon$ is very small, Eq. (6) suggests that $\tau_{\text {top }}$ is proportional to $\epsilon$ and hence is also small. $\tau_{\text {top }}$ can therefore be fully balanced by $\tau_{\text {bottom }}$ till the $1^{\text {st }}$ critical point, $\tau_{\text {top }}(a)=\tau_{0}$, where $\tau_{0}$ is the NM-substrate adhesion strength as shown in Fig. 1d 
and is hence the maximum possible $\tau_{\text {bottom }}$. The condition $\tau_{\text {top }}(a)=\tau_{0}$ corresponds to the $1^{\text {st }}$ critical loading $\epsilon_{1}=\tau_{0} h /(2 E a)$, before which no membrane stress or displacement can develop in the NM, i.e., when $0 \leq \epsilon \leq \epsilon_{1}$,

$u(x)=\sigma(x)=0(0 \leq x \leq a)$

When $\epsilon_{1}<\epsilon \leq \epsilon_{2}$, where $\epsilon_{2}$ is the $2^{\text {nd }}$ critical loading to be determined later, $\tau_{t o p}$ gets too large to be balanced by $\tau_{0}$, and hence displacement and membrane stress in the NM emerge in Zone $A C$ (Fig. 2a). The corresponding displacement and membrane stress are found to be

\begin{tabular}{|l|l|l|}
\hline$u(x)=\left\{\begin{array}{cc}0 & \left(0 \leq x \leq x_{1}\right) \\
\frac{\left(x-x_{1}\right)^{2}}{\left(2 \mu_{m}+\lambda_{m}\right) h_{m}}\left[\frac{\tau_{0}}{2}-\frac{E \epsilon}{3 h}\left(x+2 x_{1}\right)\right] & \left(x_{1}<x \leq a\right)\end{array}\right.$ & (10) \\
\hline$\sigma(x)=\left\{\begin{array}{cc}0 & \left(0 \leq x \leq x_{1}\right) \\
\left(x-x_{1}\right)\left[\tau_{0}-\frac{E \epsilon}{h}\left(x+x_{1}\right)\right] & \left(x_{1}<x \leq a\right)\end{array}\right.$ \\
\hline
\end{tabular}

where $x_{1} \in(0, a)$ represents the location of Point $A$ in Fig. 2a-2c, which can be obtained from the traction free $\mathrm{BC} \sigma(a)=0$ :

$x_{1}=\frac{\tau_{0} h}{E \epsilon}-a$

Figures $2 \mathrm{~b} \& 2 \mathrm{c}$ plot Eqs. (10) \& (11), respectively. Starting from Point $A$, although $u(x)$ is monotonically increasing till reaching the maximum at $x=a($ Fig. 2b), $\sigma(x)$ is proportional to the first derivative of $u(x)$, which is non-monotonic and maximizes somewhere within Zone $A C$ (Fig. 2c). During this time, the maximum NM-substrate separation $u(a)$ is still below the critical separation defined in the TSR (Fig. 1d), i.e. $u(a)<\delta_{c}$, which means the NM over Zone $A C$ is bounded to the substrate with partial damage. 
Further increasing $\epsilon$ would arrive at the $2^{\text {nd }}$ critical point when $\tau_{\text {bottom }}=\tau_{0}$ is reached along the entire NM-substrate interface (Fig. 2 d), i.e. $x_{1}=0$, which yields the $2^{\text {nd }}$ critical loading $\epsilon_{2}=\tau_{0} h /(E a)$. Note that $\epsilon_{1}=0.5 \epsilon_{2}$. If $u(a)=\delta_{c}$ is also reached at this critical point, then $\delta_{c}=\delta_{0}$, where

$$
\delta_{0}=\frac{\tau_{0} a^{2}}{6\left(2 \mu_{m}+\lambda_{m}\right) h_{m}}
$$

is a characteristic length scale of the NM-substrate system, which is independent of the stamp. Taking CVD monolayer graphene on seed $\mathrm{Cu}$ as an example, $h_{m}=$ $0.34 \mathrm{~nm}, \mu_{m}=427.4 \mathrm{GPa}$ and $\lambda_{m}=220.2 \mathrm{GPa}$ can be calculated from the Young's modulus (1 Ta [32]) and Poisson's ratio (0.17 [33]) of graphene. According to a recent double cantilever adhesion test [34], CVD graphene to seed $\mathrm{Cu}$ adhesion energy is measured to be $\Gamma=6 \mathrm{~J} / \mathrm{m}^{2}$ with an ultra-long range interaction of $\delta_{c}=$ $4 \mu \mathrm{m}$. Assuming a rectangular TSR, we can estimate $\tau_{0} \sim \Gamma / \delta_{c}$ to be $1.5 \mathrm{MPa}$. With a graphene flake of size $a=100 \mu \mathrm{m}, \delta_{0}$ is found to be $6.8 \mu \mathrm{m}$, which can be close to $\delta_{c}$. Figures $2 \mathrm{e}-2 \mathrm{f}$ plot the displacement, and membrane stress in the NM when $\epsilon=\epsilon_{0}$ by setting $x_{1}=0$ in Eqs. (10) \& (11).

Now let's use the ratio between the critical separation $\delta_{c}$ and the system length scale $\delta_{0}$, denoted as $m=\delta_{c} / \delta_{0}$, to categorize the different situations of this problem. For $m=1$, when $\epsilon>\epsilon_{2}$, fully damaged interface propagates from $C$ to $B$ (Figs. $2 \mathrm{~g}$ - $2 \mathrm{i}$ ) and the corresponding $\tau_{\text {bottom }}=0$ is drawn as red dashed line in Fig. $2 \mathrm{~g}$. The displacement and membrane stress can be found as 


\begin{tabular}{|l|ll|}
\hline$u(x)= \begin{cases}\frac{x}{x_{2}} \delta_{0}+\frac{x\left(x-x_{2}\right)}{\left(2 \mu_{m}+\lambda_{m}\right) h_{m}}\left[\frac{\tau_{0}}{2}-\frac{E \epsilon}{3 h}\left(x+x_{2}\right)\right] & \left(0 \leq x \leq x_{2}\right) \\
\frac{x}{x_{2}} \delta_{0}+\frac{x_{2}\left(x-x_{2}\right)}{\left(2 \mu_{m}+\lambda_{m}\right) h_{m}}\left[\frac{\tau_{0}}{2}-\frac{E \epsilon}{3 h} \frac{x}{x_{2}}\left(x+x_{2}\right)\right] & \left(x_{2}<x \leq a\right)\end{cases}$ \\
\hline$\sigma(x)= \begin{cases}\frac{\tau_{0} a^{2}}{6 x_{2} h_{m}}+\frac{1}{h_{m}}\left[\frac{\tau_{0}}{2}\left(2 x-x_{2}\right)-\frac{E \epsilon}{3 h}\left(3 x^{2}-x_{2}^{2}\right)\right]\left(0 \leq x \leq x_{2}\right) \\
\frac{\tau_{0} a^{2}}{6 x_{2} h_{m}}+\frac{1}{h_{m}}\left[\frac{\tau_{0}}{2} x_{2}-\frac{E \epsilon}{3 h}\left(3 x^{2}-x_{2}^{2}\right)\right]\end{cases}$
\end{tabular}

where $x_{2} \in(0, a)$ is the position of Point $B$ in Figs. $2 \mathrm{~g}-2 \mathrm{i}$, which like $x_{1}$ is also determined by the traction free $\mathrm{BC} \sigma(a)=0$ :

$\frac{E \epsilon}{\tau_{0} h}=\frac{a^{2}+3 x_{2}^{2}}{2 x_{2}\left(3 a^{2}-x_{2}^{2}\right)}$

Figures $2 \mathrm{~h} \& 2 \mathrm{i}$ plot representative displacement and membrane stress when $\epsilon>\epsilon_{2}$.

In this case, $\sigma(x)$ shows a kink at Point $B$ where there is sudden change of $\tau_{\text {bottom }}$ and the $\max (\sigma)$ always occurs at this kink.

If further increasing $\epsilon$ to approach infinity, the entire NM-substrate interface would completely fail, i.e., $x_{2}=0$, which yields the following result:

\begin{tabular}{|l|l||l|}
\hline$u(x)=\frac{E \epsilon}{3\left(2 \mu_{m}+\lambda_{m}\right) h h_{m}} x\left(3 a^{2}-x^{2}\right)(0 \leq x \leq a)$ & (17) \\
\hline$\sigma(x)=\frac{E \epsilon}{h h_{m}}\left(a^{2}-x^{2}\right)(0 \leq x \leq a)$ & (18) \\
\hline
\end{tabular}

So far, all the results are given for the scenario $m=1$ for simplicity. Results for arbitrary $m>0$ are summarized in Appendix with non-dimensionalized parameters:

$\hat{x}=x / a, \hat{u}=u / \delta_{0}$, and $\hat{\sigma}=\sigma h_{m} /\left(\tau_{0} a\right)$.

The applied compressive load $\epsilon$ is reformulated as $k=\epsilon / \epsilon_{2}$. Similar results for compressible stamps are summarized with the same non-dimensionalization in the supplementary material. 


\section{Discussion}

Since the failure criterion of brittle $\mathrm{NM}$ is simply $\max (\sigma)=\sigma_{\mathrm{cr}}$, we would like to extract $\max (\sigma)$ from the solutions provided in Section 3 (Eqs. (11) and (15)) and discuss its evolution with the applied compressive load $\epsilon$. Normalized $\max (\sigma)$ are plotted against $\epsilon / \epsilon_{2}$ in Figs. $3 \& 4$.

All the results presented so far are under the assumption that the stamp has the same lateral dimension with the NM. To account for different NM and stamp sizes, from now on we use $2 a$ to denote stamp size and $2 b$ the NM width. Hence the stamp-NM size ratio can be written as $\beta=b / a$, which in general may be any positive number. But in reality, the stamp size is most often as large as or larger than the NM size, so we will only discuss $\beta=1$ and $0<\beta<1$ cases in 4.1 and 4.2 , respectively.

\subsection{When $\mathrm{NM}$ size $=$ stamp size (i.e., $\beta=1$ )}

When NM is as large as the stamp, i.e., the edge of the NM aligns with that of the stamp, Figure 3a plots normalized $\max (\sigma)$ as a function of $\epsilon / \epsilon_{0}$ when $a / h=5$ is fixed but $m$ (i.e., $\delta_{c} / \delta_{0}$ ) varies. Let's first focus on the results of $m=1$. The behavior of the maximum stress in the NM can be categorizes into three stages which well corresponds to the analysis in Section 3: In Stage I $\left(0 \leq \epsilon / \epsilon_{2} \leq 0.5\right), \epsilon$ is too small to produce any stress in NM so $\max (\sigma)=0$ and there is no displacement in the NM; in Stage II $\left(0.5<\epsilon / \epsilon_{2} \leq 1\right)$ as represented by Eqs. (10) \& (11) and Figs. 2a-2c, nonzero displacement and stress emerge in $\mathrm{NM}$ and $\max (\sigma)$ increases with $\epsilon$ monotonically, but the displacement is smaller than the critical separation so the NM is bonded to the substrate with some partial interface damage; when it comes to Stage 
III $\left(\epsilon / \epsilon_{2}>1\right)$ as represented by Eqs. (14) \& (15) and Figs. 2g-2i, part of the NMsubstrate interface becomes fully damaged and a sudden jump of $\max (\sigma)$ is visible at $\epsilon / \epsilon_{2}=1$. The red dashed line in Fig. 3a plots

$\max (\sigma)=\frac{E a^{2}}{h h_{m}} \epsilon$

which is obtained by setting $x=0$ in Eq. (18) as well as in the Appendix (Eq. (A.11)) for the scenario of zero NM-substrate adhesion everywhere, i.e. $\tau_{\text {bottom }} \equiv 0$. Neglecting interface re-adhesion, as $\epsilon / \epsilon_{2}$ keeps increasing, the fully damaged zone keeps growing, thus the black curve will eventually converge to the red dashed line.

To look into the sudden jump of $\max (\sigma)$ at $\epsilon_{2}, \sigma(x)$ is plotted in Fig. 3b where the black curve represents $\sigma$ when $\epsilon=\epsilon_{2}$ (Eq. (11) when $x_{1}=0$ ) and the red curve plots $\sigma$ when $\epsilon \rightarrow \epsilon_{2}^{+}$(Eq. (15) when $x_{2}=a$ ). No solution is available within the area confined between the black and red curves if monotonically increasing $\epsilon$ is considered. The sudden change of $\max (\sigma)$ from the black curve to the red curve in Fig. 3b explains the jump of $\max (\sigma)$ at $\epsilon / \epsilon_{2}=1$ in Fig. 3a.

When $m$ (i.e., $\delta_{c} / \delta_{0}$ ) differs from 1 , the results are offered in the Appendix and the $\max (\sigma) \sim \epsilon$ relations are also plotted in Fig. 3a (e.g., $m=0.5$ and 1.5). It is evident that $\max (\sigma)$ behaves similarly under different $m$ but the abrupt jump of $\max (\sigma)$ occurs at smaller $\epsilon$ when $m$ is smaller. This makes sense because smaller $m$ indicates smaller $\delta_{c}$, which means the interface is more prone to complete damage. In reality, $m$ can be much larger or smaller than 1 because $\delta_{c}$ and $\delta_{0}$ can be differed by orders of magnitude. Figure 3a suggests that when $m \gg 1$, the NM-substrate interface hardly fails hence Stage III is pushed way back and the $\max (\sigma)$ has to follow the 
Stage II black curve closely. When $m \ll 1$, the NM-substrate interface is so weak that Stage III starts at very small $\epsilon$ and $\max (\sigma)$ has to follow the red dashed line (Eq. (20)) closely.

Given the fracture criterion of the $\mathrm{NM}, \max (\sigma)=\sigma_{\mathrm{cr}}$, the critical compressive load can be found through Fig. 3a by finding the intersections of the black dashed line and the black curves. Beyond this critical load the NM will rupture due to stamping.

\subsection{When NM size $<$ stamp size (i.e., $0<\beta<1$ )}

When the width of the NM $(2 b)$ is smaller than that of the stamp (2a), due to the negligible thickness and the significantly larger stiffness of the NM compared with the stamp, the shear stress at the bottom of the stamp doesn't change but only shear stress within the region of $|x| \leq b$ is exerted on the NM. Therefore the substitution $a \rightarrow b$ has to be applied to Eqs. (3) and (6) to properly describe the stamp-NM interface shear stress. Assuming the traction free boundary condition of the NM still holds as the stamp expands laterally, the BVP when $0<\beta<1$ remains the same and hence all the results in the main text and Appendix have the same expressions as long as we take the following substitutions: $a \rightarrow b, \delta_{0} \rightarrow \delta_{0}^{\prime}, m \rightarrow m^{\prime}, \hat{x} \rightarrow \hat{x}^{\prime}, \hat{u} \rightarrow \hat{u}^{\prime}$, $\hat{\sigma} \rightarrow \hat{\sigma}^{\prime}$ and $k \rightarrow k^{\prime}$, where

$$
\begin{aligned}
& \delta_{0}^{\prime}=\frac{\tau_{0} b^{2}}{6\left(2 \mu_{m}+\lambda_{m}\right) h_{m}}, m^{\prime}=\delta_{c} / \delta_{0}^{\prime} \\
& \hat{x}^{\prime}=x / b, \hat{u}=u / \delta_{0}^{\prime}, \hat{\sigma}^{\prime}=\sigma h_{f} /\left(\tau_{0} b\right) \\
& k=\epsilon / \epsilon_{2}^{\prime} \text { and } \epsilon_{2}^{\prime}=\tau_{0} h /(E b)
\end{aligned}
$$

To recover the results under the same non-dimensionalization given by Eq. (19), we just need to plug results with "prime" into Eq. (22): 
\begin{tabular}{|l|l|}
$\hat{u}=\hat{u}^{\prime} \frac{\delta_{0}^{\prime}}{\delta_{0}}=\hat{u}^{\prime} \beta^{2}, \hat{\sigma}=\beta \hat{\sigma}^{\prime}$, \\
$k=k^{\prime} / \beta$, and $m=m^{\prime} \frac{\delta_{0}^{\prime}}{\delta_{0}}$
\end{tabular}

Figure 4 plots normalized $\tau_{\text {top }}$ and $\max (\sigma)$ for different stamp-NM size combinations. In Figs. 4a \& 4b, NM size is fixed $(b / h=5)$ but stamp size is changing $\left(a_{1} / h=5\right.$ vs. $\left.a_{2} / h=10\right)$. In Fig. $4 \mathrm{a}$, the solid curve represents the $\tau_{\text {top }}$ exerted on the NM and the dashed curve represents the shear stress induced by the larger stamp outside of the NM zone. The two stamps, though of different sizes, always induce the same $\tau_{\text {top }} /(E \epsilon)$ at the stamp-NM interface $(|x / h| \leq b / h)$, which is no longer true if the stamps are made of compressible materials, such as those plotted in Fig. 1e whose $v=0.45$. Since $\tau_{\text {top }}$ exerted on the NM is the same for stamps with different $a / h$, the NM would exhibit the same $\max (\sigma) \sim \epsilon$ relation, as plotted in Fig. $4 \mathrm{~b}$ where $a_{1}$ is used to normalize the results: $\hat{\sigma}=\sigma h_{f} /\left(\tau_{0} a_{1}\right)$ and $\epsilon_{2}=\tau_{0} h /\left(E a_{1}\right)$. Thus, for incompressible stamps, changing the aspect ratio $a / h$ does not affect stress in the NM during the stamping process.

In Figs. $4 \mathrm{c} \& 4 d$, stamp size is fixed $(a / h=5)$, but NM size is changing $\left(b_{1} / h=\right.$ 5 vs. $\left.b_{2} / h=4\right)$. In Fig. $4 \mathrm{c}$, the solid curve represents the interface shear stress applied on the NM with $b_{2} / h=4$ while the solid and dashed curves together represent the interface shear stress applied on the NM with $b_{1} / h=5$. As the larger NM is subjected to higher $\tau_{\text {top }}$ near the edge, the larger NM tends to have higher membrane stress as shown in Fig. 4d. Therefore, smaller NMs are less prone to rupture under the same applied load $\epsilon$. More generically, smaller $b / h$ is effective in reducing $\max (\sigma)$. Since we already concluded from Figs. $4 \mathrm{a} \& 4 \mathrm{~b}$ that $a / h$ doesn't 
affect the NM stress for incompressible stamps, the other way to lower $b / h$ is to simply use a thicker stamp (i.e., larger $h$ ).

\section{Conclusions}

When using stamps to pick up high quality NMs from their donor substrates, stamping induced rupture becomes a dominant failure mode as the NM thickness drops to nanometers. We applied the analytical solutions we previously obtained for elastic layers compressed between parallel plates as the stamp-NM interface shear stress and analytically solved a BVP to yield the membrane stress distribution in the NM. The membrane stress depends on not only the applied load, the stamp and NM geometry and property, but also the NM-substrate interaction, which is assumed to be a rectangular TSR. We therefore found several ways to limit the maximum membrane stress in the NM: first the stamp cannot be compressed too much; second, the NM size should be small; and third, the thicker stamps are preferred. Although results in the main text are obtained for incompressible stamp material, results for compressible materials are offered in the supplementary material.

\section{Acknowledge}

This work is based upon work supported primarily by the National Science Foundation under Cooperative Agreement No. EEC-1160494. Any opinions, findings and conclusions or recommendations expressed in this material are those of the author(s) and do not necessarily reflect the views of the National Science 
Foundation. S. Q. acknowledges the John and Mary Wheeler endowed graduate fellowship.

\section{Appendix}

This Appendix solves Eq. (8) for arbitrary $m>0$ with dimensionless parameters defined in Eq. (19) and $k=\epsilon / \epsilon_{2} \cdot m=\delta_{c} / \delta_{0}$ is the ratio between the critical separation at the NM-substrate interface and the intrinsic length scale of the NM-substrate system as defined in Eq. (13). Although $\delta_{c}$ and $\delta_{0}$ are similar in the example of CVD graphene on seed $\mathrm{Cu}$ as mentioned in the main text, in general they can be differed by orders of magnitude. So here, assuming NM is as large as the stamp $(\beta=1)$, we offer the detailed dimensionless solutions for three different scenarios: $m=1, m>1$ and $0<m<1$.

1. $m=1$

\begin{tabular}{|c|c|}
\hline$\hat{u}(\hat{x})=\hat{\sigma}(\hat{x})=0(0 \leq \hat{x} \leq 1) \quad$ when $0 \leq k \leq 0.5$ & (A.1) \\
\hline $\begin{array}{l}\hat{u}(\hat{x})=\left\{\begin{array}{cc}0 & \left(0 \leq \hat{x} \leq \hat{x}_{1}\right) \\
\left(\hat{x}-\hat{x}_{1}\right)^{2}\left[3-2 k\left(\hat{x}+2 \hat{x}_{1}\right)\right]\left(\hat{x}_{1}<\hat{x} \leq 1\right)\end{array}\right. \\
\hat{\sigma}(\hat{x})=\left\{\begin{array}{cc}0 & \left(0 \leq \hat{x} \leq \hat{x}_{1}\right) \\
\left(\hat{x}-\hat{x}_{1}\right)\left(1-k \hat{x}_{1}-k \hat{x}\right) & \left(\hat{x}_{1}<\hat{x} \leq 1\right)\end{array} \text { when } 0.5<k \leq 1\right.\end{array}$ & (A.2) \\
\hline $\begin{array}{l}\hat{u}(\hat{x})= \begin{cases}\frac{\hat{x}}{\hat{x}_{2}}+3 \hat{x}\left(\hat{x}-\hat{x}_{2}\right)-2 k \hat{x}\left(\hat{x}^{2}-\hat{x}_{2}^{2}\right)\left(0 \leq \hat{x} \leq \hat{x}_{2}\right) \\
\frac{\hat{x}}{\hat{x}_{2}}+3 \hat{x}_{2}\left(\hat{x}-\hat{x}_{2}\right)-2 k \hat{x}\left(\hat{x}^{2}-\hat{x}_{2}^{2}\right)\left(\hat{x}_{2}<\hat{x} \leq 1\right)\end{cases} \\
\hat{\sigma}(\hat{x})=\left\{\begin{array}{ll}\frac{1}{6 \hat{x}_{2}}+\left(\hat{x}-\frac{\hat{x}_{2}}{2}\right)-k\left(\hat{x}^{2}-\frac{\hat{x}_{2}^{2}}{3}\right) & \left(0 \leq \hat{x} \leq \hat{x}_{2}\right) \\
\frac{1}{6 \hat{x}_{2}}+\frac{\hat{x}_{2}}{2}-k\left(\hat{x}^{2}-\frac{\hat{x}_{2}^{2}}{3}\right) & \left(\hat{x}_{2}<\hat{x} \leq 1\right)\end{array} \text { when } k>1\right.\end{array}$ & (A.3) \\
\hline
\end{tabular}

where $\hat{x}_{1}=1 / k-1, \hat{x}_{2}$ is given by the traction free boundary condition, $\hat{\sigma}(1)=0$ and is reformulated as: 


\begin{tabular}{|l|l|l|}
\hline$k=\frac{1+3 \hat{x}_{2}^{2}}{2 \hat{x}_{2}\left(3-\hat{x}_{2}^{2}\right)}$ & (A.4) \\
\hline
\end{tabular}

2. $m>1$ :

When $0 \leq k \leq 0.5$ and $0.5<k \leq 1$, solutions are the same as Eqs. (A.1) and (A.2), respectively. Otherwise, solutions are given by the following equations:

\begin{tabular}{|c|}
\hline $\begin{array}{l}\hat{u}(\hat{x})=3 \hat{x}(\hat{x}-2)-2 k \hat{x}\left(\hat{x}^{2}-3\right)(0 \leq \hat{x} \leq 1) \\
\hat{\sigma}(\hat{x})=(1-\hat{x})(k \hat{x}+k-1) \quad(0 \leq \hat{x} \leq 1)\end{array} \quad$ when $1<k \leq k_{1}$ \\
\hline $\begin{array}{l}\hat{u}(\hat{x})=\left\{\begin{array}{l}\frac{m \hat{x}}{\hat{x}_{2}}+3 \hat{x}\left(\hat{x}-\hat{x}_{2}\right)-2 k \hat{x}\left(\hat{x}^{2}-\hat{x}_{2}^{2}\right)\left(0 \leq \hat{x} \leq \hat{x}_{2}\right) \\
\frac{m \hat{x}}{\hat{x}_{2}}+3 \hat{x}_{2}\left(\hat{x}-\hat{x}_{2}\right)-2 k \hat{x}\left(\hat{x}^{2}-\hat{x}_{2}^{2}\right)\left(\hat{x}_{2}<\hat{x} \leq 1\right)\end{array} \quad \text { when } k>k_{1}\right. \\
\hat{\sigma}(\hat{x})= \begin{cases}\frac{m}{6 \hat{x}_{2}}+\left(\hat{x}-\frac{\hat{x}_{2}}{2}\right)-k\left(\hat{x}^{2}-\frac{\hat{x}_{2}^{2}}{3}\right) & \left(0 \leq \hat{x} \leq \hat{x}_{2}\right) \\
\frac{m}{6 \hat{x}_{2}}+\frac{\hat{x}_{2}}{2}-k\left(\hat{x}^{2}-\frac{\hat{x}_{2}^{2}}{3}\right) & \left(\hat{x}_{2}<\hat{x} \leq 1\right)\end{cases} \end{array}$ \\
\hline
\end{tabular}

where $k_{1}$ is given by the condition $\left.\hat{u}(1)\right|_{k=k_{1}}=m$ and turns out to be

$k_{1}=\frac{m+3}{4}$

and $\hat{x}_{2}$ is the root of $\hat{\sigma}(1)=0$ in $(0,1)$ and, similarly to Eq. (A.4), is rewritten as

$k=\frac{m+3 \hat{x}_{2}^{2}}{2 \hat{x}_{2}\left(3-\hat{x}_{2}^{2}\right)}$.

3. $0<m<1$ :

When $0 \leq k \leq 0.5$, the solution is the same as Eq. (A.1). When $0.5<k \leq k_{2}$, the solution is the same with Eq. (A.2), and $k_{2}$ is obtained from the condition $\left.\hat{u}(1)\right|_{k=k_{2}}=m$ together with $\hat{x}_{1}=1 / k-1$. After simplification, we can get Eq. (A.9) from which $k_{2}$ could be solved for:

$m=k_{2}\left(2-1 / k_{2}\right)^{3}$

Besides, when $k>k_{2}$, the solution is the same with Eq. (A.6). 
If there is no interface adhesion, i.e. $\tau_{\text {bottom }} \equiv 0$, the solution is:

\begin{tabular}{|l|l|l|}
\hline$\hat{u}(\hat{x})=2 k\left(3 \hat{x}-\hat{x}^{3}\right)(0 \leq \hat{x} \leq 1)$ & (A.10) \\
$\hat{\sigma}(\hat{x})=k\left(1-\hat{x}^{2}\right)(0 \leq \hat{x} \leq 1)$ & \\
\hline
\end{tabular}

and the corresponding maximum membrane stress is

$$
\max (\hat{\sigma})=\hat{\sigma}(0)=k
$$

\section{References}

[1] Park, S. I., Xiong, Y. J., Kim, R. H., Elvikis, P., Meitl, M., Kim, D. H., Wu, J., Yoon, J., Yu, C. J., Liu, Z. J., Huang, Y. G., Hwang, K., Ferreira, P., Li, X. L., Choquette, K., and Rogers, J. A., 2009, "Printed Assemblies of Inorganic Light-Emitting Diodes for Deformable and Semitransparent Displays," Science, 325(5943), pp. 977-981.

[2] Yoon, J., Baca, A. J., Park, S. I., Elvikis, P., Geddes, J. B., Li, L. F., Kim, R. H., Xiao, J. L., Wang, S. D., Kim, T. H., Motala, M. J., Ahn, B. Y., Duoss, E. B., Lewis, J. A., Nuzzo, R. G., Ferreira, P. M., Huang, Y. G., Rockett, A., and Rogers, J. A., 2008, "Ultrathin silicon solar microcells for semitransparent, mechanically flexible and microconcentrator module designs," Nat Mater, 7(11), pp. 907-915.

[3] Yoon, J., Jo, S., Chun, I. S., Jung, I., Kim, H. S., Meitl, M., Menard, E., Li, X. L., Coleman, J. J., Paik, U., and Rogers, J. A., 2010, "GaAs photovoltaics and optoelectronics using releasable multilayer epitaxial assemblies," Nature, 465(7296), pp. 329-333.

[4] Kim, R. H., Kim, D. H., Xiao, J. L., Kim, B. H., Park, S. I., Panilaitis, B., Ghaffari, R., Yao, J. M., Li, M., Liu, Z. J., Malyarchuk, V., Kim, D. G., Le, A. P., Nuzzo, R. G., Kaplan, D. L., Omenetto, F. G., Huang, Y. G., Kang, Z., and Rogers, J. A., 2010, "Waterproof AlInGaP optoelectronics on stretchable substrates with applications in biomedicine and robotics," Nat Mater, 9(11), pp. 929-937.

[5] Lee, J., Wu, J. A., Shi, M. X., Yoon, J., Park, S. I., Li, M., Liu, Z. J., Huang, Y. G., and Rogers, J. A., 2011, "Stretchable GaAs Photovoltaics with Designs That Enable High Areal Coverage," Adv Mater, 23(8), pp. 986-991.

[6] Ko, H. C., Stoykovich, M. P., Song, J. Z., Malyarchuk, V., Choi, W. M., Yu, C. J., Geddes, J. B., Xiao, J. L., Wang, S. D., Huang, Y. G., and Rogers, J. A., 2008, "A hemispherical electronic eye camera based on compressible silicon optoelectronics," Nature, 454(7205), pp. 748-753.

[7] Song, Y. M., Xie, Y., Malyarchuk, V., Xiao, J., Jung, I., Choi, K. J., Liu, Z., Park, H., Lu, C., Kim, R. H., Li, R., Crozier, K. B., Huang, Y., and Rogers, J. A., 2013, "Digital cameras with designs inspired by the arthropod eye," Nature, 497(7447), pp. 95-99.

[8] Kim, D. H., Ghaffari, R., Lu, N. S., and Rogers, J. A., 2012, "Flexible and Stretchable Electronics for Bio-Integrated Devices," Annual review of biomedical engineering, 14, pp. 113128.

[9] Kim, D. H., Lu, N. S., Ghaffari, R., and Rogers, J. A., 2012, "Inorganic semiconductor nanomaterials for flexible and stretchable bio-integrated electronics," Npg Asia Mater, 4, p. e15. [10] Kim, D. H., Lu, N. S., Huang, Y. G., and Rogers, J. A., 2012, "Materials for stretchable electronics in bioinspired and biointegrated devices," Mrs Bull, 37(3), pp. 226-235. 
[11] Bruel, M., 1995, "Silicon-on-Insulator Material Technology," Electron Lett, 31(14), pp. 1201-1202.

[12] Ploog, K., 1981, "Molecular-Beam Epitaxy of Iii-V Compounds - Technology and GrowthProcess," Annu Rev Mater Sci, 11, pp. 171-210.

[13] Meitl, M. A., Zhu, Z. T., Kumar, V., Lee, K. J., Feng, X., Huang, Y. Y., Adesida, I., Nuzzo, R. G., and Rogers, J. A., 2006, "Transfer printing by kinetic control of adhesion to an elastomeric stamp," Nat Mater, 5(1), pp. 33-38.

[14] Feng, X., Meitl, M. A., Bowen, A. M., Huang, Y., Nuzzo, R. G., and Rogers, J. A., 2007, "Competing fracture in kinetically controlled transfer printing," Langmuir, 23(25), pp. 1255512560.

[15] Carlson, A., Bowen, A. M., Huang, Y. G., Nuzzo, R. G., and Rogers, J. A., 2012, "Transfer Printing Techniques for Materials Assembly and Micro/Nanodevice Fabrication," Adv Mater, 24(39), pp. 5284-5318.

[16] Ahn, J. H., Kim, H. S., Lee, K. J., Zhu, Z. T., Menard, E., Nuzzo, R. G., and Rogers, J. A., 2006, "High-speed mechanically flexible single-crystal silicon thin-film transistors on plastic substrates," Ieee Electr Device L, 27(6), pp. 460-462.

[17] Service, R. F., 2006, "Materials science - Inorganic electronics begin to flex their muscle," Science, 312(5780), pp. 1593-1594.

[18] Schmidt, O. G., and Eberl, K., 2001, "Nanotechnology - Thin solid films roll up into nanotubes," Nature, 410(6825), pp. 168-168.

[19] Zhang, L., Ruh, E., Grutzmacher, D., Dong, L. X., Bell, D. J., Nelson, B. J., and Schonenberger, C., 2006, "Anomalous coiling of SiGe/Si and SiGe/Si/Cr helical nanobelts," Nano Lett, 6(7), pp. 1311-1317.

[20] Yuan, H. C., Shin, J. H., Qin, G. X., Sun, L., Bhattacharya, P., Lagally, M. G., Celler, G. K., and Ma, Z. Q., 2009, "Flexible photodetectors on plastic substrates by use of printing transferred single-crystal germanium membranes," Appl Phys Lett, 94(1).

[21] Ko, H., Takei, K., Kapadia, R., Chuang, S., Fang, H., Leu, P. W., Ganapathi, K., Plis, E., Kim, H. S., Chen, S. Y., Madsen, M., Ford, A. C., Chueh, Y. L., Krishna, S., Salahuddin, S., and Javey, A., 2010, "Ultrathin compound semiconductor on insulator layers for high-performance nanoscale transistors," Nature, 468(7321), pp. 286-289.

[22] Kim, T. H., Carlson, A., Ahn, J. H., Won, S. M., Wang, S. D., Huang, Y. G., and Rogers, J. A., 2009, "Kinetically controlled, adhesiveless transfer printing using microstructured stamps," Appl Phys Lett, 94(11).

[23] Kim, S., Wu, J. A., Carlson, A., Jin, S. H., Kovalsky, A., Glass, P., Liu, Z. J., Ahmed, N., Elgan, S. L., Chen, W. Q., Ferreira, P. M., Sitti, M., Huang, Y. G., and Rogers, J. A., 2010, "Microstructured elastomeric surfaces with reversible adhesion and examples of their use in deterministic assembly by transfer printing," P Natl Acad Sci USA, 107(40), pp. 17095-17100.

[24] Green, M. A., and Keevers, M. J., 1995, "Optical-Properties of Intrinsic Silicon at 300 K," Prog Photovoltaics, 3(3), pp. 189-192.

[25] Hwang, S. W., Tao, H., Kim, D. H., Cheng, H. Y., Song, J. K., Rill, E., Brenckle, M. A., Panilaitis, B., Won, S. M., Kim, Y. S., Song, Y. M., Yu, K. J., Ameen, A., Li, R., Su, Y. W., Yang, M. M., Kaplan, D. L., Zakin, M. R., Slepian, M. J., Huang, Y. G., Omenetto, F. G., and Rogers, J. A., 2012, "A Physically Transient Form of Silicon Electronics," Science, 337(6102), pp. 1640-1644.

[26] Hwang, S. W., Lee, C. H., Cheng, H. Y., Jeong, J. W., Kang, S. K., Kim, J. H., Shin, J., Yang, J., Liu, Z. J., Ameer, G. A., Huang, Y. G., and Rogers, J. A., 2015, "Biodegradable Elastomers and Silicon Nanomembranes/Nanoribbons for Stretchable, Transient Electronics, and Biosensors," Nano Lett, 15(5), pp. 2801-2808.

[27] Seo, J. H., Chang, T. H., Lee, J., Sabo, R., Zhou, W. D., Cai, Z. Y., Gong, S. Q., and Ma, Z. Q., 2015, "Microwave flexible transistors on cellulose nanofibrillated fiber substrates," Appl Phys Lett, 106(26). 
[28] Brennan, C. J., Nguyen, J., Yu, E. T., and Lu, N., 2015, "Interface Adhesion between 2D Materials and Elastomers Measured by Buckle Delaminations," Advanced Materials Interfaces, pp. n/a-n/a.

[29] Qiao, S., and Lu, N., 2015, "Analytical Solutions for Bonded Elastically Compressible Layers," Int J Solids Struct, 58, pp. 353-365.

[30] Gent, A., and Lindley, P., 1959, "The compression of bonded rubber blocks," Proceedings of the Institution of Mechanical Engineers, 173(1), pp. 111-122.

[31] Gent, A., Henry, R., and Roxbury, M., 1974, "Interfacial stresses for bonded rubber blocks in compression and shear," Journal of Applied Mechanics, 41(4), pp. 855-859.

[32] Lee, C., Wei, X. D., Kysar, J. W., and Hone, J., 2008, "Measurement of the elastic properties and intrinsic strength of monolayer graphene," Science, 321(5887), pp. 385-388.

[33] Liu, F., Ming, P. M., and Li, J., 2007, "Ab initio calculation of ideal strength and phonon instability of graphene under tension," Phys Rev B, 76(6).

[34] Na, S. R., Suk, J. W., Tao, L., Akinwande, D., Ruoff, R. S., Huang, R., and Liechti, K. M., 2015, "Selective Mechanical Transfer of Graphene from Seed Copper Foil Using Rate Effects," Acs Nano, 9(2), pp. 1325-1335.

\section{Figure Captions}

Figure 1 (a) A stamp (pink) backed by a rigid layer (blue) is just in contact with a NM (red) bonded on its donor substrate (green). The Young's modulus, Poisson's ratio and thickness of the stamp are $E, v$, and $h$ respectively. The Lamé constants and thickness of the NM are $\mu_{\mathrm{m}}, \lambda_{\mathrm{m}}$, and $h_{\mathrm{m}}$, respectively. The stamp and NM have the same width of $2 a$. (b) The stamp is compressed vertically and hence expands laterally, which applies shear stress on the top surface of the NM that may result in NM rupture. (c) Stresses applied to the right half of the NM. $\tau_{\text {top }}$ is applied by the stamp and $\tau_{\text {bottom }}$ is applied by the donor substrate. The inset shows a free body diagram of the boxed part of the NM. (d) A rectangular traction separation relation (TSR) is assumed for the NM-substrate interface. $\tau_{0}$ is the adhesion strength and $\delta_{c}$ is the critical sepeartion. (e) The distribution of normalized $\tau_{\text {top }}$ when two stamps have the same Poisson's ratio $(v=0.45)$ but different aspect ratios (red: $a / h=8$ vs. black: $a / h=10$ ). (f) The distribution of normalized $\tau_{\text {top }}$ when stamps have the same aspect ratio $(a / h=5)$ but different Poisson's ratios $(v=$ $0.3,0.48,0.495$ and 0.5$)$. 
Figure 2 (a)-(c) The applied shear stresses, displacement, and membrane stress distribution in the NM when $\epsilon / \epsilon_{2}=0.8$ where $\epsilon_{2}=\tau_{0} h /(E a) . \tau_{\text {bottom }}$ reaches $\tau_{0}$ in Zone $A C$, which is a partially damaged NM-substrate interface. (d)-(f) $\epsilon / \epsilon_{2}=1$, which is the critical moment before the NM-substrate interface starts to have fully damaged zone. (g)-(i) $\epsilon / \epsilon_{2}=1.3$, where NM is fully detached from the substrate in Zone $B C$ and hence $\tau_{\text {bottom }}=0$ in Zone $B C$.

Figure 3 (a) Normalized $\max (\sigma)$ vs. $\epsilon / \epsilon_{2}$ for $m=0.5,1$, and 1.5 . The abrupt jump corresponds to the sudden failure of the NM-substrate interface when the critical separation is first reached. (b) Normalized $\sigma(x)$ at $\epsilon=\epsilon_{2}$ (black curve) and $\epsilon \rightarrow \epsilon_{2}^{+}$(red curve) show a sudden transition. No solutions are available between the curves.

Figure 4 (a) Distribution of normalized $\tau_{\text {top }}$ with fixed NM size $b / h$ and two different stamp sizes: $a_{1} / h=5$ and $a_{2} / h=10$. The $\tau_{\text {top }}$ applied on the NM is given by the solid curve which is the same under different $a / h$. (b) Normalized max $(\sigma)$ in the NM is the same when only $a / h$ is changing. (c) Distribution of normalized $\tau_{t o p}$ with fixed stamp size $a / h$ and two different NM sizes: $b_{1} / h=5$ and $b_{2} / h=4$. Solid curve represents the $\tau_{\text {top }}$ applied on the smaller NM whereas solid and dashed curves together represent the $\tau_{\text {top }}$ applied on the larger NM. (d) Normalized $\max (\sigma)$ is higher for systems with larger $b / h$. 
Fig. 1

(a) Rigid backing layer

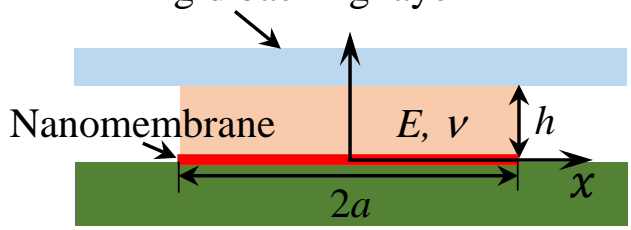

(c)

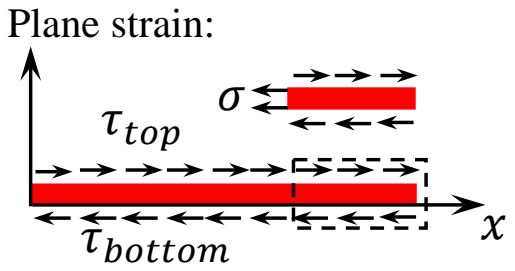

(e)

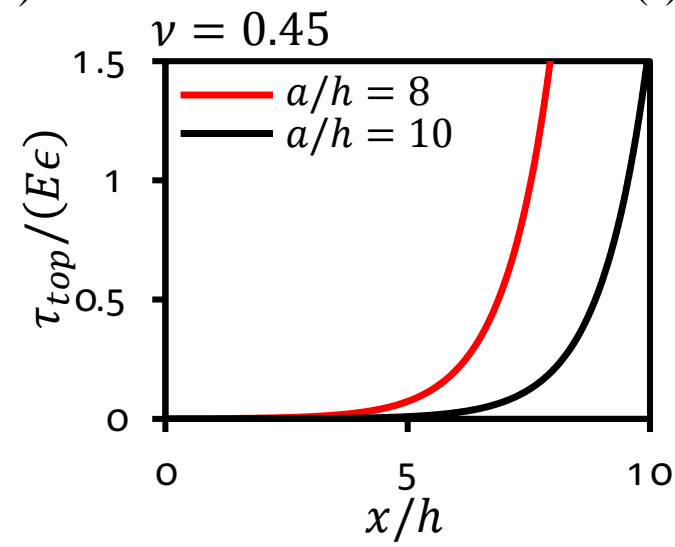

(b)

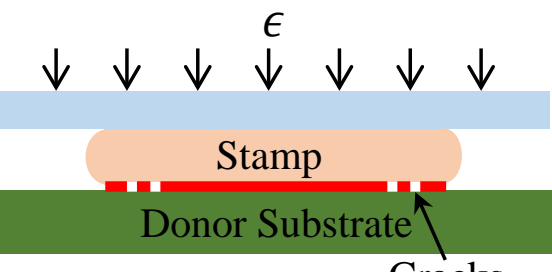

(d)
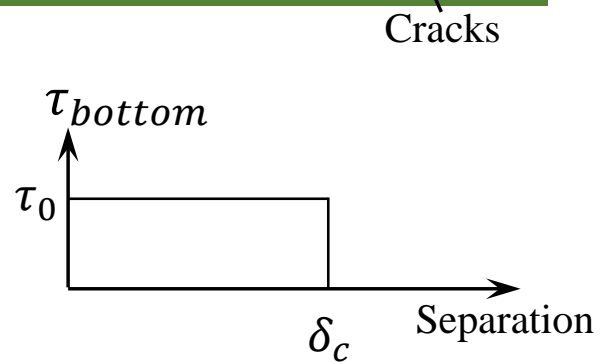

(f)

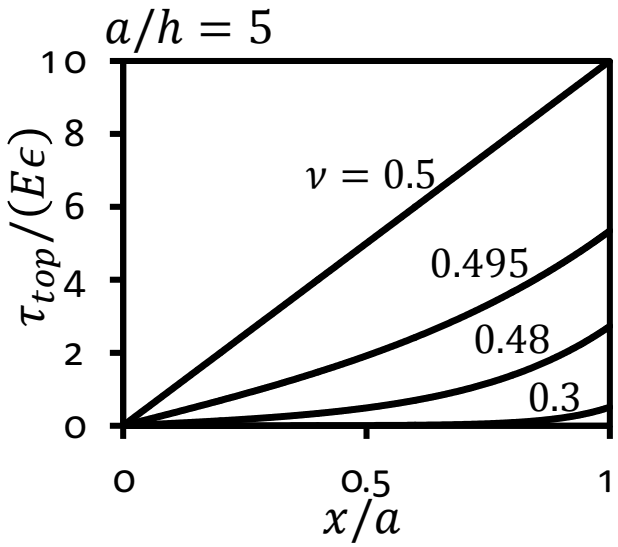


Fig. 2
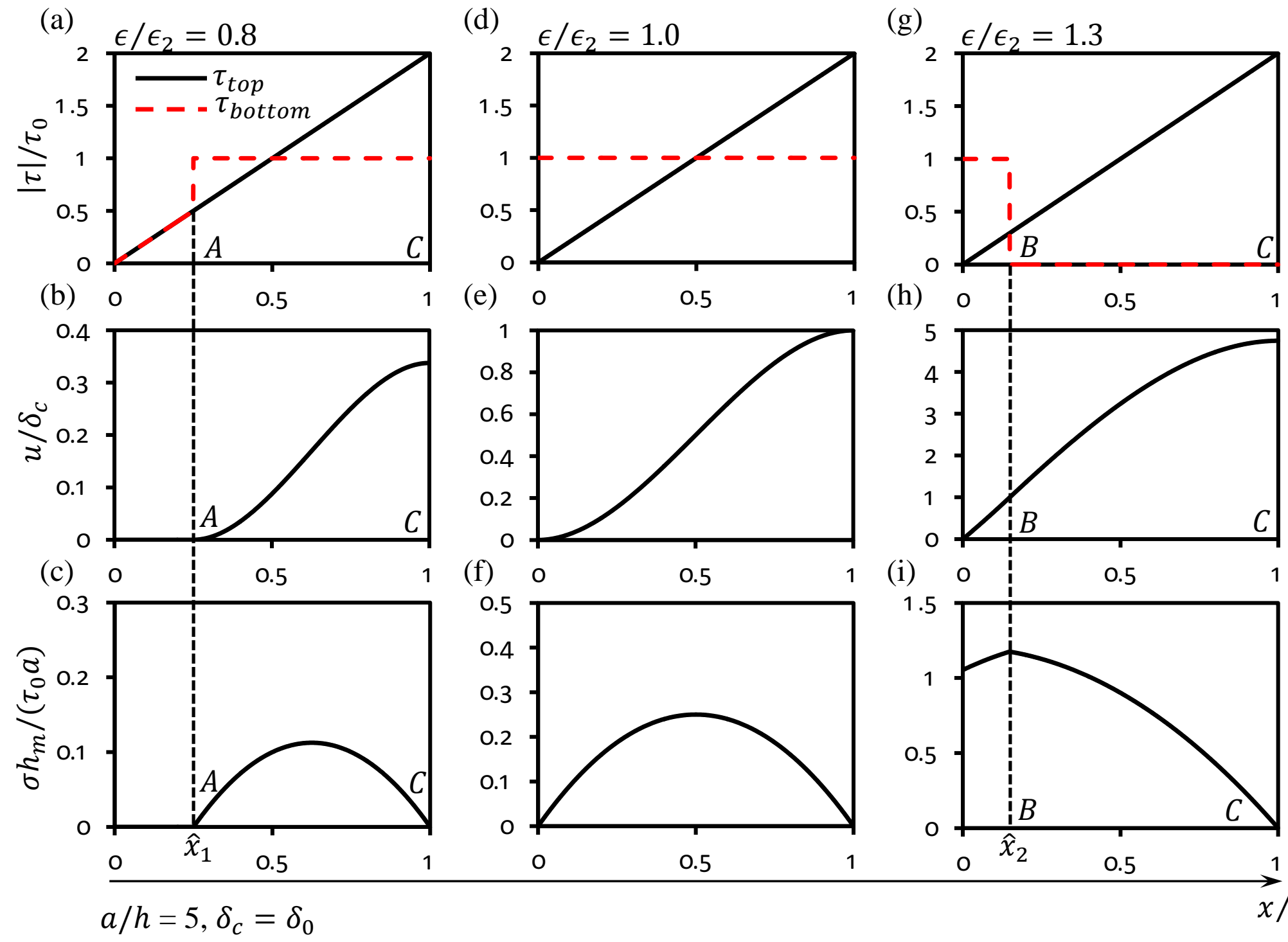

(i)
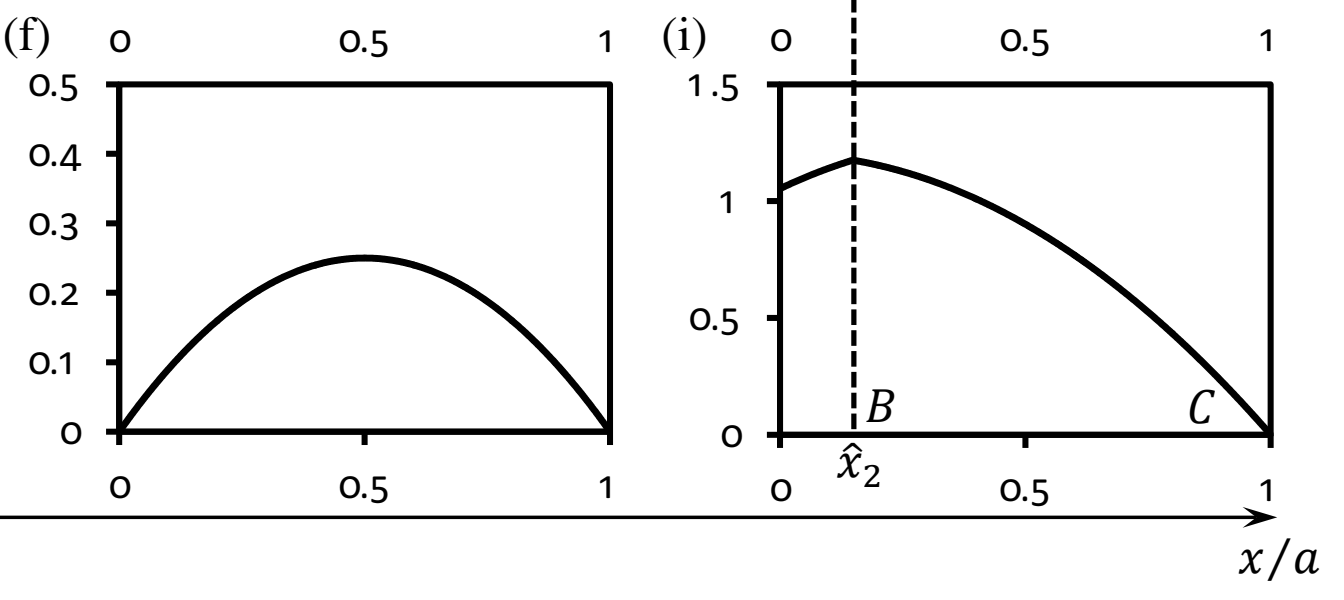
Fig. 3

(a) $a / h=5, \delta_{c}=\delta_{0}$

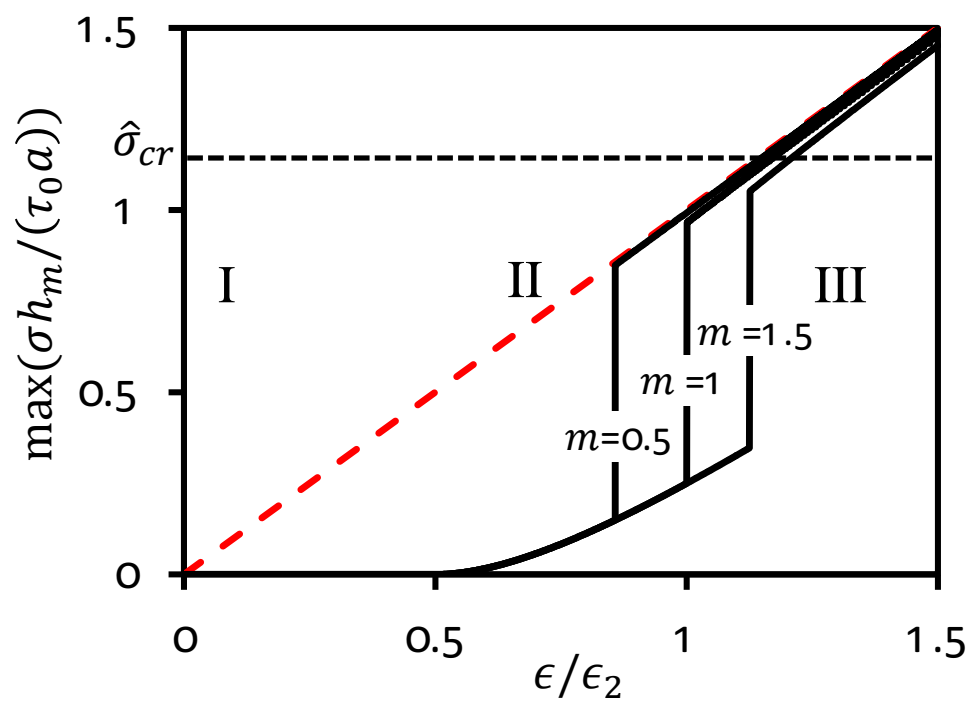

(b)

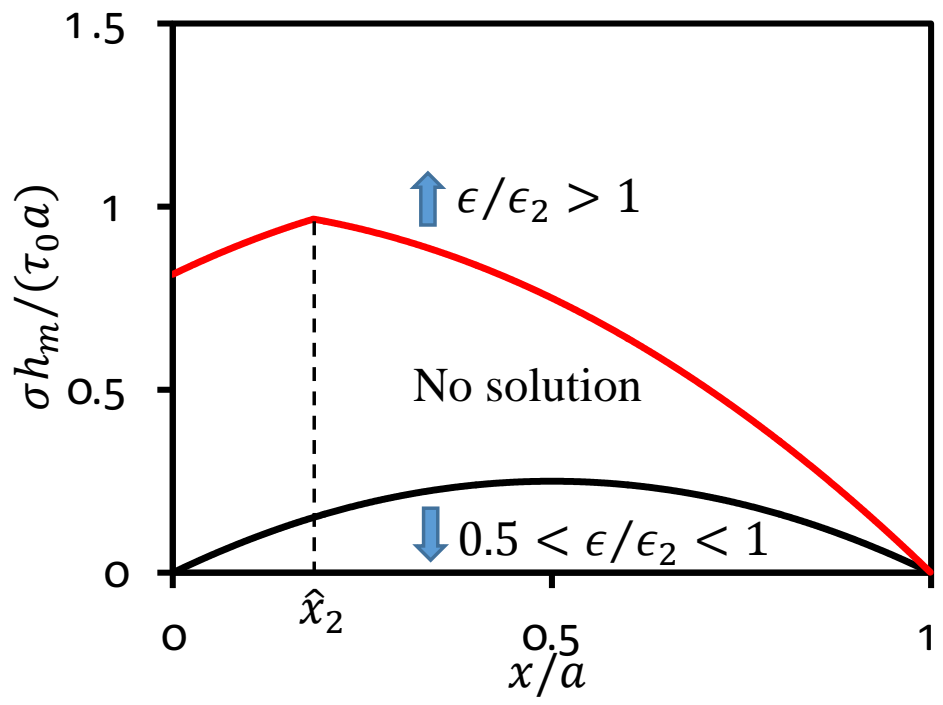


Fig. 4

(a)

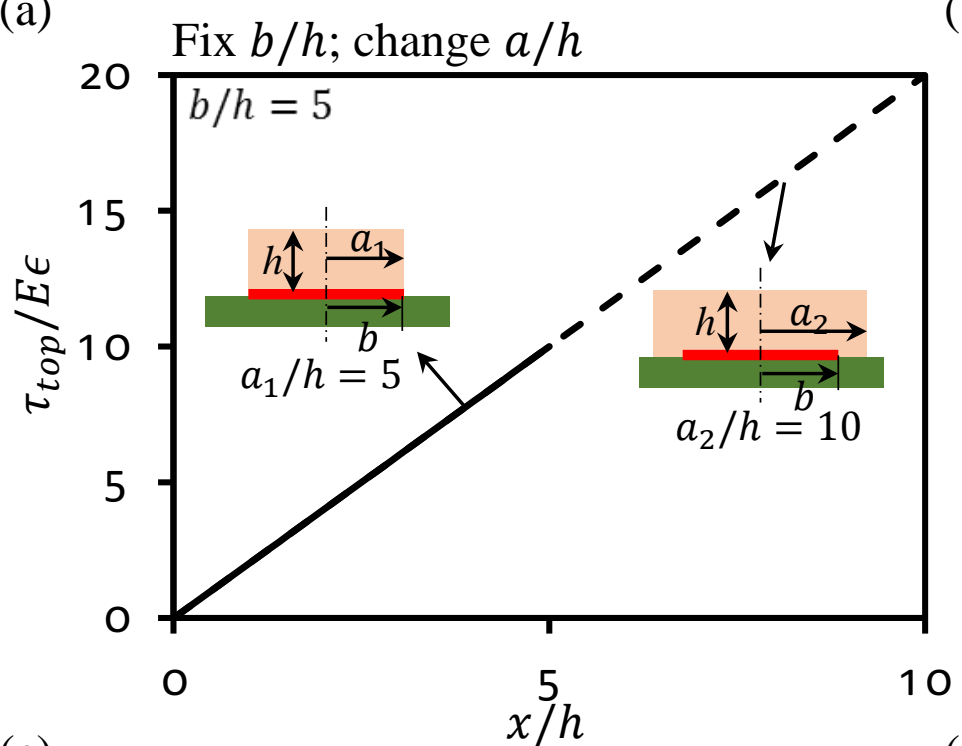

(c) Fix $a / h$; change $b / h$

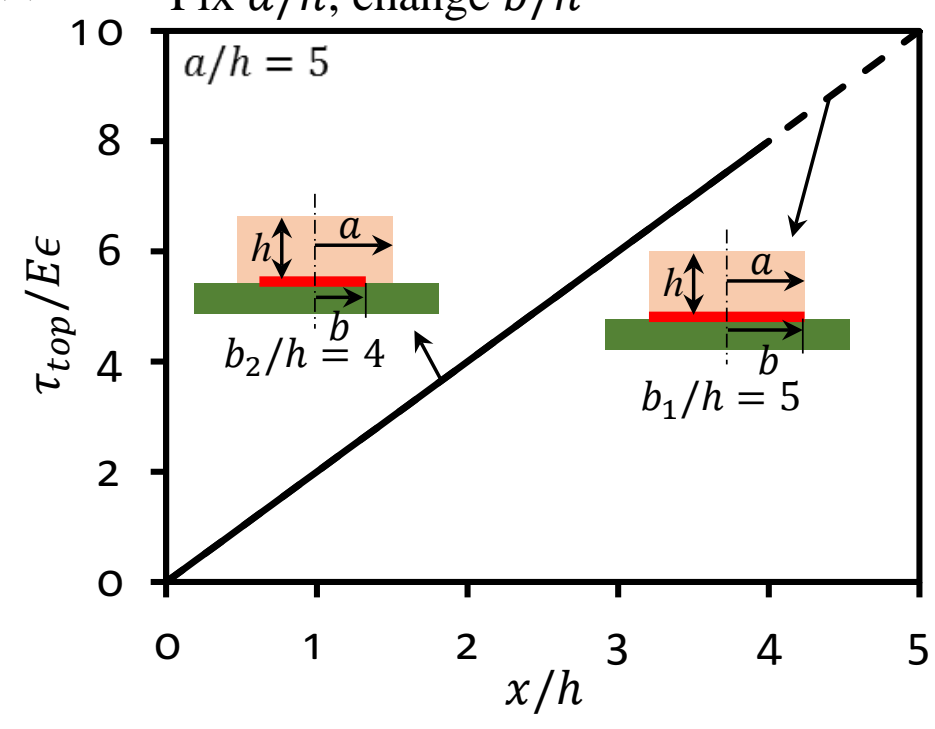

(b)

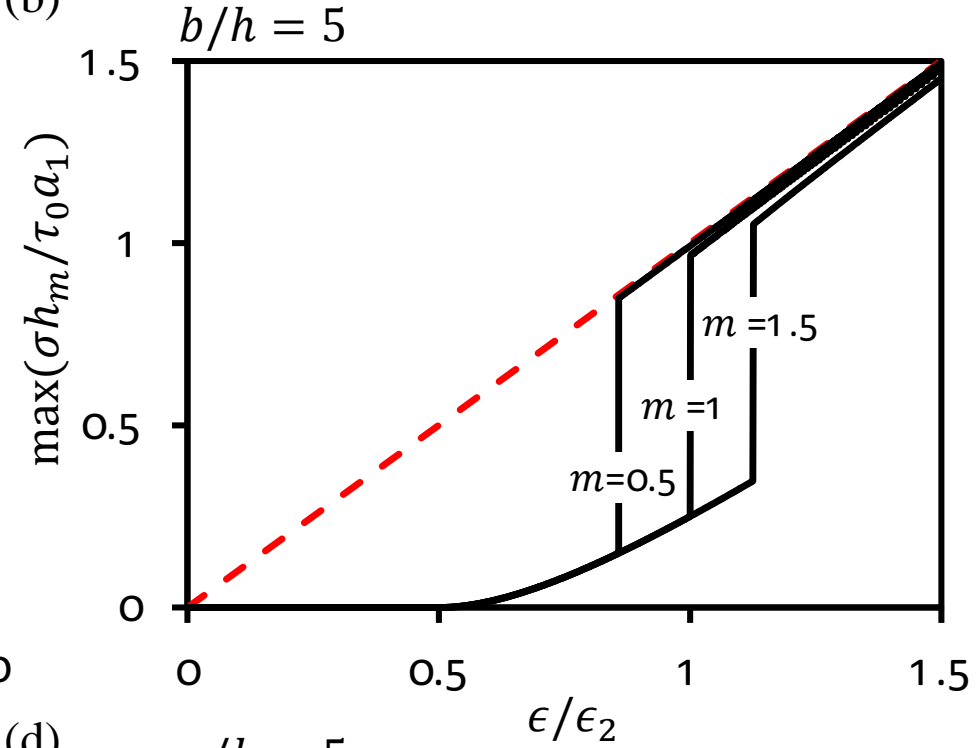

(d)

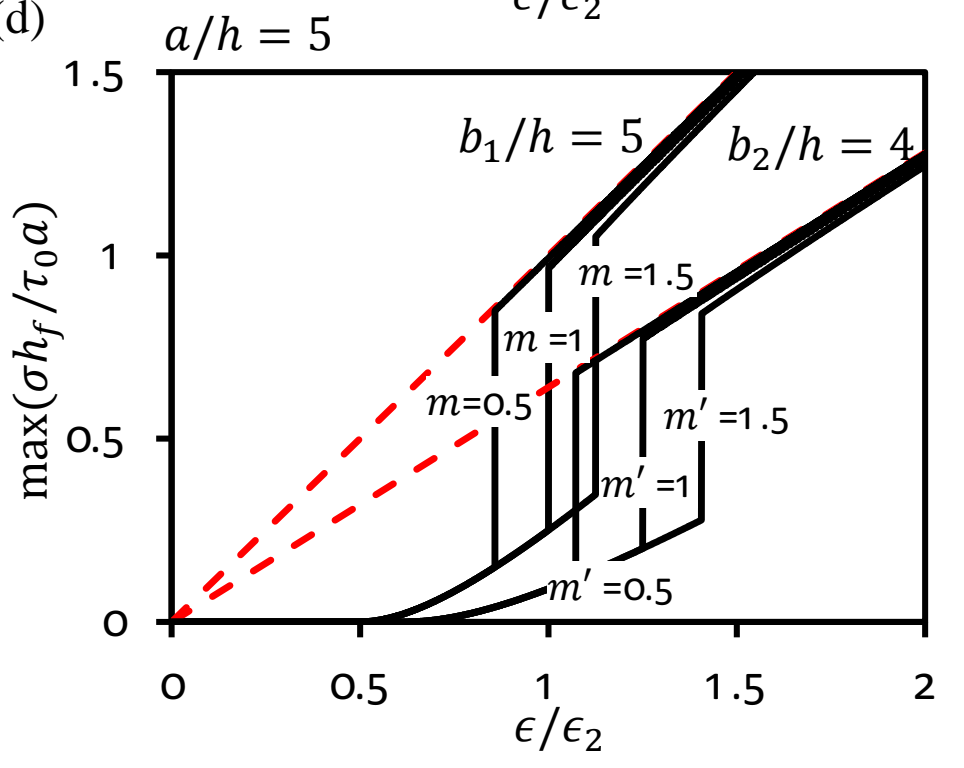

